\title{
ENTEROPATÓGENOS DE IMPORTANCIA EN SALUD PÚBLICA
}

\author{
RELEVANT PUBLIC HEALTH ENTEROPATHOGENS \\ Maribel Riveros ${ }^{1, a}$, Theresa J. Ochoa ${ }^{1,2, b}$ \\ Universidad Peruana Cayetano Heredia, Lima, Perú. \\ Center for Infectious Diseases, School of Public Health, University of Texas Health Science Center at Houston, Texas, Estados Unidos. \\ a Licenciado en Biología; ${ }^{b}$ médico pediatra infectólogo. \\ Recibido: 13-02-15; Aprobado: 18-03-15
}

\begin{abstract}
RESUMEN
La diarrea continúa siendo la tercera causa de muerte en niños menores de 5 años, pese a los avances recientes en el manejo y prevención de esta enfermedad. Es causada por múltiples patógenos, sin embargo, la prevalencia de cada uno varía según el grupo de edad, la zona geográfica y el escenario donde se registran los casos (comunitario vs hospitalario). Los patógenos de mayor relevancia en salud pública son aquellos asociados con mayor carga de enfermedad, severidad, complicaciones y mortalidad. En nuestro medio, el norovirus, Campylobacter y las $E$. coli diarrogénicas, son los patógenos más prevalentes a nivel comunitario en niños. En este artículo se revisa la epidemiología local y las potenciales áreas de desarrollo en cinco patógenos seleccionados: rotavirus, norovirus, E. coli productora de toxina Shiga (STEC), Shigella y Salmonella. De estos, el rotavirus es el más importante en la población pediátrica y el principal responsable de la mortalidad infantil por diarrea. La introducción de la vacunación contra rotavirus en nuestro país tendrá un importante impacto en la carga de enfermedad y la mortalidad por diarrea. Sin embargo, se requieren estudios de vigilancia para determinar el impacto de la vacunación y el cambio en la epidemiología de la diarrea en el Perú luego de la introducción de nuevas vacunas, así como la vigilancia de las tasas de resistencia antibiótica para las bacterias de importancia clínica.
\end{abstract}

Palabras clave: Escherichia coli enteropatógena; Diarrea; Niño; Rotavirus; Norovirus; Escherichia coli enterohemorrágica (fuente: DeCS BIREME).

\begin{abstract}
Diarrhea remains the third leading cause of death in children under five years, despite recent advances in the management and prevention of this disease. It is caused by multiple pathogens, however, the prevalence of each varies by age group, geographical area and the scenario where cases (community vs hospital) are recorded. The most relevant pathogens in public health are those associated with the highest burden of disease, severity, complications and mortality. In our country, norovirus, Campylobacter and diarrheagenic E. coli are the most prevalent pathogens at the community level in children. In this paper we review the local epidemiology and potential areas of development in five selected pathogens: rotavirus, norovirus, Shiga toxin-producing E. coli (STEC), Shigella and Salmonella. Of these, rotavirus is the most important in the pediatric population and the main agent responsible for child mortality from diarrhea. The introduction of rotavirus vaccination in Peru will have a significant impact on disease burden and mortality from diarrhea. However, surveillance studies are needed to determine the impact of vaccination and changes in the epidemiology of diarrhea in Peru following the introduction of new vaccines, as well as antibiotic resistance surveillance of clinical relevant bacteria.
\end{abstract}

Key words: Enteropathogenic Escherichia coli; Diarrhea; Child; Rotavirus; Norovirus; Enterohemorrhagic Escherichia coli (source: MeSH NLM).

\section{INTRODUCCIÓN}

La diarrea continúa siendo una las principales causas de morbi-mortalidad, principalmente en niños. Pese a los avances en las últimas décadas en el manejo y prevención de diarrea (uso de suero de baja osmolaridad, uso de zinc, desarrollo de nuevas vacunas), esta sigue siendo la tercera causa de muerte en menores de 5 años. En una análisis global para el 2011, se estimó que aproximadamente 7,6 millones de niños mueren cada año, de los cuales $10,41 \%$ son por diarrea $(0,8$ millones de muertes) ${ }^{(1)}$.

Además de los efectos en mortalidad, la diarrea infantil afecta el crecimiento y desarrollo. Los niños con episodios prolongados de diarrea o episodios a

Citar como: Riveros M, Ochoa TJ. Enteropatógenos de importancia en salud pública. Rev Peru Med Exp Salud Publica. 2015;32(1):157-64. 
repetición tienen mayor probabilidad de desarrollar no solo desnutrición aguda (adelgazamiento), sino también desnutrición crónica (talla baja). Los episodios de diarrea a repetición en los primeros dos años de vida se correlacionan con menor neurodesarrollo, bajo rendimiento escolar y salarios menos remunerados en la edad adulta.

Los patógenos asociados con diarrea incluyen virus (rotavirus, norovirus, astrovirus y adenovirus entéricos), bacterias (Shigella, Salmonella, Campylobacter, Vibrio, E. coli diarrogénica, Aeromonas, Yersinia, entre otros), y parásitos (Cryptosporidum, Giardia, Cyclospora, Microsporidium, Entamoeba histolytica, Balantidium coli). La frecuencia relativa de cada patógeno varía dependiendo del grupo de edad, lugar y si el registro de casos es a nivel comunitario u hospitalario. Los estudios comunitarios pueden ser considerados un proxy de morbilidad, mientras que los hospitalarios, por seleccionar a los casos más severos, podrían considerarse un proxy de mortalidad.

Un estudio reciente diseñado para determinar la etiología y carga de diarrea en países en vías de desarrollo (The Global Enteric Multicenter Study, GEMS), evaluó más de 9000 casos de diarrea moderada/severa en niños menores de 5 años y en más de 13000 niños sin diarrea (control), en cuatro países de África y tres de Asia ${ }^{(2)}$. La mayoría de los casos de diarrea moderada/severa fueron causados por cuatro patógenos: rotavirus, Cryptosporidium, E. coli enterotoxigénica con toxina termoestable (ETEC-ST) y Shigella. Los niños con diarrea moderada/severa tuvieron 8,5 veces más riesgo de morir hasta los 60 días de seguimiento; E. coli enteropatógena (EPEC), Cryptosporidium, y ETEC-ST fueron los patógenos asociados con mayor mortalidad.

El grupo de investigación del Laboratorio de Enfermedades Entéricas y Nutrición (LEEN), del Instituto de Medicina Tropical Alexander von Humboldt de la Universidad Peruana Cayetano Heredia realizó dos estudios comunitarios para determinar la prevalencia de patógenos entéricos asociados con diarrea en niños menores de 2 años en zonas periurbanas de Lima. El primer estudio se hizo en cuatro distritos del cono sur (Chorrillos, Villa El Salvador, Villa María de Triunfo y San Juan de Miraflores) entre los años 2006-2007. Se siguieron 1034 niños desde los 2 a los 12 meses de edad. Se realizó una vigilancia "pasiva"; se pidió a las madres, cuyos hijos participaban en un estudio de vacunas, que acudieran al consultorio del estudio si su niño presentaba diarrea ${ }^{(3)}$. Se encontró una incidencia de 0,98 episodios por niño por año. El segundo estudio fue parte de un ensayo clínico diseñado para determinar el efecto de lactoferrina en la prevención de diarrea en 555 niños entre 12 y 24 meses de edad. Se realizó en el distrito de Independencia entre los años 2008-2011. Cada niño fue seguido durante 6 meses con visitas domiciliarias diarias para identificar todos los episodios de diarrea ${ }^{(4)}$. En el primer estudio la incidencia de diarrea fue de 0,98 episodios por niño por año, mientras que en el segundo fue de 5,2 episodios por niño por año. Lo cual era de esperar, por ser el segundo una vigilancia activa. La búsqueda de patógenos se hizo de manera sistemática en todas las muestras, utilizando la misma metodología en ambos estudios, excepto por la falta de búsqueda de norovirus en el primer estudio. Los principales patógenos aislados en ambos estudios fueron las E. coli diarrogénicas y Campylobacter, mientras que norovirus fue el patógeno más frecuentemente aislado en el segundo estudio, con más de un tercio de las muestras positivas para este germen (Tabla 1).

Tabla 1. Distribución de patógenos entéricos en niños menores de 2 años con diarrea en comunidades urbanomarginales de Lima.

\begin{tabular}{|c|c|c|}
\hline $\begin{array}{l}\text { Patógeno } \\
\text { entérico }\end{array}$ & $\begin{array}{c}\text { Cohorte 1- } \\
\text { Lima Sur } \\
1034 \text { niños }<12 \\
\text { meses } \\
\text { (n=936 muestras) * }\end{array}$ & $\begin{array}{c}\text { Cohorte 2- } \\
\text { Lima Norte } \\
555 \text { niños } 12-24 \\
\text { meses } \\
(n=915 \text { muestras })^{\star *}\end{array}$ \\
\hline & $\mathrm{n}(\%)$ & $\mathrm{n}(\%)$ \\
\hline \multicolumn{3}{|l|}{ Bacteria } \\
\hline Campylobacter & $174(18,6)$ & $97(10,6)$ \\
\hline Shigella & $1(0,1)$ & $60(6,6)$ \\
\hline Vibrio & $0(0)$ & $2(0,2)$ \\
\hline Otras bacterias $†$ & & $10(1,1)$ \\
\hline EPEC & $71(7,6)$ & $104(11,4)$ \\
\hline$E A E C$ & $141(15,1)$ & $76(8,4)$ \\
\hline ETEC & $30(3,2)$ & $63(6,9)$ \\
\hline DAEC & $43(4,6)$ & $27(3,0)$ \\
\hline EIEC & $0(0)$ & $7(0,8)$ \\
\hline STEC & $5(0,5)$ & $5(0,5)$ \\
\hline \multicolumn{3}{|l|}{ Virus } \\
\hline Rotavirus & $161(17,2)^{£}$ & $26(3,9)$ \\
\hline Adenovirus & - & $46(3,7)$ \\
\hline Norovirus & $39(17,4)^{*}$ & $275(35,0)$ \\
\hline \multicolumn{3}{|l|}{ Parásitos } \\
\hline Giardia & - & $58(6,3)$ \\
\hline Blastocystis & - & $17(1,9)$ \\
\hline Cryptosporidium & - & $5(0,6)$ \\
\hline Cyclospora & - & $2(0,2)$ \\
\hline Strongyloides & - & $1(0,1)$ \\
\hline Otros parásitos $\ddagger$ & - & $29(3,3)$ \\
\hline
\end{tabular}

*Modificado de Ochoa, 2009; ** Modificado de Ochoa, 2013 ; †Otras bacterias: Aeromonas, Plesiomonas, Salmonella. ₹ Slo se analizó rotavirus en 851 muestras; ${ }^{*}$ Slo se analizó norovirus en 224 muestras; ‡Otros parásitos: Chilomastix, Endolimax, Entamoeba, Enterobius, Diphyllobothrium, Hymenolepis, Trichuris, Isospora.

$\mathrm{DAEC}, E$. coli de adherencia difusa; EAEC, E. coli enteroagregativa; EIEC, E. coli enteroinvasiva; EPEC, E. coli enteropatogénica; ETEC, E. coli enterotoxigénica; STEC, E. coli productora de toxina Shiga. 
Los patógenos de mayor relevancia en salud pública son los asociados con mayor carga de enfermedad, severidad, complicaciones y mortalidad. Si bien el norovirus, Campylobacter y las E. coli diarrogénicas en conjunto, son los patógenos más prevalentes a nivel comunitario en nuestro medio, consideramos que otros patógenos son también relevantes, sobre todo por el mayor riesgo de complicaciones y mortalidad. Revisemos algunos de estos patógenos.

\section{ROTAVIRUS}

Es el patógeno más importante en la población pediátrica y el principal responsable de la mortalidad infantil por diarrea. El rotavirus produce una diarrea acuosa abundante, altamente deshidratante acompañada por vómitos y fiebre (triada diagnóstica clásica). Un estudio de 10 años realizado en nuestro país antes de la introducción de la vacuna en el Programa Nacional de Inmunizaciones estimó que para los 5 años de edad, más del $63 \%$ de la población había tenido, al menos, un episodio de diarrea por rotavirus; 1 en 10 buscaron atención medica en consulta externa; 1 en 20 fueron hospitalizados, y 1 en 375 fallecieron ${ }^{(5)}$. La vacuna se introdujo al esquema nacional de inmunizaciones entre los años 2008-2009. Estudios posteriores, basados en las actuales guías de la Organización Mundial de la Salud (OMS), han demostrado que la vacunación contra rotavirus es una intervención altamente costo-efectiva en el Perú ${ }^{(6)}$. Sin embargo, la vacuna contra rotavirus disminuye las tasas de hospitalización, enfermedad severa y muerte, pero no elimina la infección leve/ moderada. Porlotanto, pese a la introducción de la vacuna y su impacto en la mortalidad y carga de enfermedad, aún seguiremos viendo casos de diarrea por rotavirus. El rotavirus presenta una estacionalidad característica, por lo que es conocido como la "diarrea del invierno". Un estudio realizado entre el 2008 y el 2011 demostró que la infección por rotavirus en niños de Lima presenta una clara estacionalidad con un pico bien marcado en los meses de mayo a agosto. Adicionalmente, se observó una disminución significativa en la fracción de episodios por rotavirus a nivel comunitario en los años siguientes a la introducción de la vacuna ${ }^{(7)}$.

El diagnóstico de rotavirus es mayormente presuntivo, en base a la edad del niño, las características clínicas y la estacionalidad. Para confirmar su diagnóstico se pueden hacer pruebas inmunológicas (detección de antígeno en heces) o moleculares (reacción en cadena de la polimerasa o PCR). La prueba inmunológica más sencilla, rápida y barata es la inmunocromatografia, sin embargo, el ELISA tiene mejor sensibilidad $y$ especificidad. Un estudio en el Hospital Nacional Arzobispo Loayza en Lima, demostró que el uso de una prueba rápida de rotavirus en la sala de emergencias en niños menores de 5 años con diarrea, disminuyó significativamente la prescripción innecesaria de antibióticos ${ }^{(8)}$. Por lo tanto, se debería implementar en los servicios de salud estrategias para el diagnóstico focalizado en el invierno y así evitar el uso inadecuado de antibióticos en la diarrea infantil.

Desde el punto de vista de la prevención, la cobertura de vacunación contra el rotavirus en el Perú para el año 2013 fue de 86,3\% (Dirección General de Estadística e Informática del MINSA). Es prioritario mejorar estas coberturas de vacunación hasta alcanzar niveles adecuados (mayor a 95\%), sobre todo en la población más vulnerable, como los bebes prematuros, quienes tienen menores tasas de vacunación y mayores $\operatorname{atrasos}^{(9)}$.

\section{NOROVIRUS}

En general, este patógeno es responsable del 20 al $30 \%$ de los casos endémicos de diarrea en niños y está asociado a brotes de gastroenteritis. En los países donde se ha introducido la vacuna contra rotavirus, el norovirus es el principal patógeno en pacientes pediátricos que buscan atención médica por gastroenteritis aguda. En un estudio realizado en Las Pampas de San Juan de Miraflores en el cono sur de Lima, se demostró que la infección por norovirus ocurre a edad temprana. El $80 \%$ de los niños han experimentado, por lo menos, una infección por norovirus al año de edad, mientras que a los dos años el $71 \%$ ha tenido, al menos, un episodio de diarrea asociado a norovirus ${ }^{(10)}$. El cuadro clínico es similar al de la infección por rotavirus; los pacientes presentan diarrea acuosa, vómitos y fiebre; sin embargo, la diarrea es usualmente de menor duración (promedio 2-3 días). En un estudio en 224 muestras de niños menores de 24 meses en el cono sur de Lima, se detectó norovirus en $17,4 \%$, siendo más frecuente en niños mayores de 12 meses (34 vs. $8 \%, p<0,001$ ). Cuando se comparó con rotavirus, el cuadro clínico de la diarrea asociada a norovirus fue de menor duración y menos severo ${ }^{(11)}$. Por otro lado, los episodios de diarrea asociados a norovirus muestran una alta prevalencia de coinfecciones con otros virus, bacterias y parásitos. En el estudio desarrollado en el distrito de Independencia (Cohorte 2, Tabla 1), se encontró que 53\% de los episodios positivos a norovirus tienen una coinfección, comparado con solo $12 \%$ en muestras negativas a norovirus. Los cuadros de diarrea por norovirus en el que hay una coinfección, ya sea con Campylobacter, 
Shigella o una E. coli diarrogénica, presentan mayor severidad clínica que las infecciones únicas por uno de estos patógenos ${ }^{(12)}$. Adicionalmente, se encontró que los casos de norovirus ocurren sin una clara estacionalidad en Lima.

Uno de los principales problemas con norovirus es el diagnóstico. Actualmente, el mejor método diagnóstico es por PCR; sin embargo, este método solo está disponible en laboratorios de investigación. Los laboratorios clínicos locales, usan el método de ELISA, el cual tiene menor sensibilidad. Por otro lado, este virus tiene un tiempo de excreción bastante prolongado, en promedio, aproximadamente 2 semanas. En el estudio de Saito en Las Pampas de San Juan de Miraflores, el tiempo de excreción de norovirus genotipo II (el genotipo más frecuente) fue de 34 días ${ }^{(10)}$. Esto dificulta el diagnóstico, pues la identificación del norovirus en un niño con diarrea, no indica necesariamente que este es el agente causante, ya que podría tratarse de una excreción prolongada de un episodio previo. Esto se corrobora por las altas tasas de infección asintomática reportada en niños, principalmente en países en vías de desarrollo. Para resolver este problema, probablemente se tengan que hacer estudios de carga viral en heces.

En cuanto a prevención, hay vacunas en fase de desarrollo que actualmente se están probando en ensayos clínicos. Una vacuna efectiva podrá tener un gran impacto en la morbilidad por norovirus.

\section{E. coli PRODUCTORA DE TOXINA SHIGA (STEC)}

También se le conoce con el nombre de E. coli enterohemorrágica (EHEC) o E. coli verotoxigénica (VTEC). Esta bacteria se caracteriza por producir la toxina llamada shiga (sxt), que puede ser de dos tipos: sxt1 y sxt2. Si bien puede estar asociada con infección asintomática y diarrea acuosa, STEC se caracteriza por producir diarrea disentérica o colitis francamente hemorrágica. Entre un 5-10\% de los pacientes infectados pueden desarrollar luego una complicación severa, el síndrome urémico hemolítico (HUS por sus siglas en inglés). Este síndrome, que ocurre mayormente en la población pediátrica, se caracteriza por anemia hemolítica intravascular, plaquetopenia y falla renal; siendo una de las principales causa de insuficiencia renal en niños. La ocurrencia de esta complicación está asociada a la sxt2 y al serotipo 0157:H7; sin embargo, en muchas partes del mundo predominan los serotipos no-O157, por lo que se debe buscar la presencia de la toxina y no solo el serotipo específico.
En el Perú se reporta la presencia de STEC mediante PCR en $0,8 \%$ (95\% IC, 0,5-1,0) de 4240 muestras de diarrea (mayoritariamente acuosas) de niños menores de 5 años, y en $0,5 \%(95 \%$ IC, 0,3-0,8) de 3760 muestras de niños sin diarrea (colonización). En una colección de 20 cepas STEC bien caracterizadas, aisladas de niños peruanos, se encontró stx 1 en 18 cepas, stx 2 en 2 , y el gen de intimina (eae) en 16. El serotipo más frecuente fue $\mathrm{O} 26: \mathrm{H} 11$, presente en 7 cepas (35\%); otros serotipos encontrados fueron el 0111, 01:H7 y O6. En el análisis filogenético (PFGE, Pulsed field gel electrophoresis), el grupo clonal más prevalente fue EHEC2 ${ }^{(13)}$. En otro estudio en el servicio de emergencia del Hospital Nacional Cayetano Heredia, en 131 pacientes menores de 5 años con disentería, encontramos STEC en 9,2\% (por PCR o ELISA) ${ }^{(14)}$. Los niños con STEC presentaron fiebre solo en un tercio de los episodios frente a dos tercios en los casos cuya disentería se debió a otro patógeno, principalmente Shigella. Este dato clínico, la ausencia o menor frecuencia de fiebre en la infección por STEC, puede ser usado como un signo de alarma para sospechar en este patógeno y pedir pruebas confirmatorias. La importancia de hacer el diagnóstico radica en la recomendación de no dar antibióticos a niños con STEC, pues concentraciones subinhibitorias del antibiótico pueden inducir la mayor síntesis de toxina Shiga $y$, por ende, mayor riesgo de desarrollar HUS.

La STEC es transmitida por alimentos o agua contaminada con heces de animales, principalmente del ganado vacuno. La carne de res puede contaminarse con la bacteria o la toxina por prácticas inadecuadas durante la matanza de estos animales. Con el objetivo de determinar la prevalencia de colonización de STEC en ganado, se realizó un estudio en diez granjas de Lima, encontrando STEC en $14 \%$ de 114 muestras de hisopado rectal en ganado vacuno ${ }^{(15)}$. En cuanto a alimentos, un estudio realizado en diferentes mercados de Lima Metropolitana, encontró a E. coli O157 en 12,3\% de 407 muestras de alimentos (23 en 102 muestras de carnes molida, 15 en carne entera, 8 en 102 muestras de queso fresco, y 2 en 101 verduras frescas) ${ }^{(16)}$.

Si bien nuestras tasas de aislamiento de STEC y la prevalencia de HUS son menores que otros países de la región, como Argentina, tenemos evidencia de que este patotipo de $E$. coli se encuentra presente en muestras de animales, alimentos y pacientes. Por lo tanto, urge establecer claras recomendaciones sanitarias para la identificación temprana de este patógeno en pacientes con sospecha clínica. Se debería establecer guías para el manejo diagnóstico de diarrea disentérica en los principales hospitales del país. El diagnóstico se debe hacer mediante ELISA, para determinar la toxina. Se pueden usar también otras pruebas rápidas como 
el látex y la inmunocromatografía, pero con menor sensibilidad/especificidad.

\section{Shigella}

Esta bacteria es la principal causa de disentería, tanto en niños como en adultos, en todo el mundo. Es responsable, aproximadamente, del $5-10 \%$ de casos de diarrea acuosa y $30 \%$ de los casos de disentería. La mayoría de las infecciones por Shigella ocurren en países en vías de desarrollo; $70 \%$ de todos los casos y $60 \%$ de todas las muertes ocurren en niños menores de 5 años. Existen cuatro especies: S. sonnei, S. boydii, S. flexneri y $S$. dysenteriae. La frecuencia de cada especie varía según la zona geográfica; en los países en vías de desarrollo, como el nuestro, es frecuente encontrar $S$. flexneri, mientras que en países desarollados, S. sonnei; sin embargo, en los últimos años se ha observado algunos cambios. Un reporte del Centro de Investigaciones Médicas de la Marina de Estados Unidos en el Perú (NAMRU-6), en una colección de 3270 aislamientos de Shigella, mostró que S. sonnei representó entre el 26$30 \%$ de todas las cepas aisladas en los años 2005-2007, mientras que en el año 2008 representó el 47\% (Bernal M, comunicación personal). Si bien esto se podría deber a un brote, es algo que se debe vigilar. Es de esperar que se incremente la proporción de casos de $S$. sonnei a mayor desarrollo económico de la población, como se ha observado en Chile y otros países de la región.

Las manifestaciones clínicas de la infección por Shigella varían dependiendo de la especie, por ejemplo $S$. dysenteriae serotipo 1 es la especie asociada a mayor severidad ya que posee la toxina shiga, responsable del síndrome urémico hemolítico (las otras especies no tienen esta toxina). S. dysenteriae es responsable de brotes de disentería severa, principalmente en Asia y África. Sin embargo, esta especie es rara en nuestro medio, encontrándose en menos del $5 \%$ de todos los casos de Shigella. Por otro lado, S. sonnei presenta un cuadro clínico más leve que $S$. flexneri (mayor proporción de diarrea acuosa).

El hombre es el principal reservorio y la vía de transmisión es fecal-oral siendo la más común de persona a persona. Es un patógeno de bajo inóculo, por lo tanto, se transmite fácilmente por las manos. En los países en vías de desarrollo, la transmisión está asociada con pobreza, falta de higiene, y consumo de agua y alimentos contaminados.

Como parte de un estudio multicéntrico que se está realizando a nivel mundial para determinar los factores de riesgo y la interacción entre las infecciones entéricas y la malnutrición (MAL-ED), se siguió una cohorte de niños en comunidades rurales de lquitos. Se encontraron altas tasas de diarrea y desnutrición crónica, donde la Shigella es el segundo agente bacteriano más común en esta población ${ }^{(17)}$. Estudios previos realizados en la misma zona encontraron altas tasas de infección por Shigella, principalmente entre los 12 y 60 meses de edad ${ }^{(18)}$.

Este patógeno debe ser tratado con antibióticos para disminuir el riesgo de complicaciones, el tiempo de enfermedad y la excreción de la bacteria en las heces, y por ende, la posibilidad de contaminación y casos secundarios. La resistencia a antimicrobianos se ha convertido en unos de los principales problemas para el tratamiento de enfermedades diarreicas, especialmente en población pediátrica. Estudios nacionales han demostrado que los perfiles de susceptibilidad varían según la especie de Shigella. En el estudio de Kosek ${ }^{(18)}$ en lquitos, en una muestra de 400 aislamientos de Shigella, $79 \%$ fueron resistentes a cotrimoxazol, $73 \%$ a ampicilina y $62 \%$ a cloranfenicol; por otro lado, la resistencia a azitromicina fue $16 \%$, a ácido nalidíxico $5 \%$ y a ciprofloxacina $3 \%$. Nuestro grupo analizó 85 cepas de niños del cono norte de Lima entre los años 20082010 encontrando $86 \%$ de resistencia a cotrimoxazol, $69 \%$ a ampicilina y $57 \%$ a cloranfenicol ${ }^{(19)}$. Por los elevados niveles de resistencia, ya no se recomienda el uso empírico de ampicilina o cotrimoxazol.

La OMS recomienda como tratamiento de primera línea, tanto para niños como adultos, la ciprofloxacina, y como segunda línea la ceftriaxona o la azitromicina. El problema actual es la emergencia de resistencia a estos "nuevos" antibióticos, sobre todo en el sur de Asia, donde se están reportando cepas resistentes a quinolonas. A nivel local se debe vigilar la resistencia al ácido nalidíxico, dado que es un "marcador" de resistencia futura a ciprofloxacina en la comunidad. En nuestro país, el Ministerio de Salud en el año 2001 en una colección de 106 cepas de Shigella encontró que el $3 \%$ presentó susceptibilidad intermedia y el $1 \%$ fue resistente al ácido nalidíxico ${ }^{(20)}$. En el año 2002 , Llance reportó $14,4 \%$ de cepas de Shigella resistentes al ácido nalidíxico y $1,8 \%$ de cepas resistentes al ciprofloxacino de un total de 111 cepas aisladas en el Hospital Nacional Daniel Alcides Carrión (21).

También están emergiendo cepas resistentes a las cefalosporinas de tercera generación, principalmente en países de África y Asia. La presencia de cepas de Shigella flexneri productoras de $\beta$-lactamasas tipo CTX-M han sido reportadas en muestras de heces de pacientes pediátricos con diarrea aguda admitidos en el servicio de emergencia del Instituto Nacional de Salud 
del Niño del Perú ${ }^{22)}$. Todos estos datos sugieren la necesidad de una estricta vigilancia de la resistencia antibiótica fenotípica y genotípica en nuestro medio, así como reforzar el uso racional de antibióticos.

Debido a que deben hacerse las elecciones de tratamiento rápidamente y los coprocultivos convencionales carecen de una buena sensibilidad, existe la necesidad de técnicas de detección rápidas, sensibles y baratas. Nuestro equipo desarrolló un protocolo de PCR en tiempo real múltiplex para identificar simultáneamente Campylobacter, Salmonella, y Shigella ${ }^{(23)}$.

Desde el punto de vista de prevención, aún no hay una buena vacuna contra Shigella para la población pediátrica. El problema radica en la gran variabilidad de serotipos en diferentes partes del mundo y la pobreprotección en niños. Hay varias vacunas en desarrollo.

\section{Salmonella}

Las bacterias del género Salmonella pueden producir gastroenteritis, fiebre tifoidea, bacteremia e infecciones localizadas como meningitis y osteomielitis. La gastroenteritis por Salmonella puede presentarse como una diarrea acuosa o disentérica. La manifestación de la enfermedad depende tanto de la susceptibilidad del huésped (edad, desnutrición, inmunosupresión, enfermedad de células falciformes, neutropenia, entre otros) y la capacidad patogénica de la bacteria. Se estima que anualmente se producen 93,8 millones de casos de Salmonella no Typhi y aproximadamente 150 000 muertes en todo el mundo ${ }^{(24)}$.

En los estudios comunitarios realizados en Lima, la prevalencia de Salmonella en casos de diarrea en niños menores de dos años, es muy baja ${ }^{(3,4)}$. Sin embargo, esta bacteria se reporta en nuestro país como causante de brotes comunitarios e intrahospitalarios. Los reportes del Instituto Nacional de Salud (INS) sobre prevalencia de patógenos entéricos muestra porcentajes elevados de Salmonella. Sin embargo, creemos que estos reportes tienen un sesgo de selección, dado que muchas cepas de Salmonella son enviadas al INS para serotipificación o para estudios de brotes. Estudios realizados en Cajamarca, Lambayeque, Loreto y Lima en muestras de diarrea en niños menores de 5 años, registraron una frecuencia de Salmonella del 13,2\%, incluyendo $15,3 \%$ en muestras de diarrea acuosa y $8,7 \%$ de diarrea disentérica ${ }^{(20)}$. En la ciudad de Loreto, en un estudio de un brote en 212 personas, ocho aislamientos fueron identificados como Salmonella enteritidis mediante tipificación serológica (somática y flagelar) y subtipificación molecular por PFGE, identificándose el mismo perfil genético para las ocho cepas procedentes del brote ${ }^{(25)}$. En el 2001 se reportó un brote intrahospitalario por Salmonella typhimurium productora de betalactamasa de espectro extendido SHV-5 en Lima ${ }^{(26)}$. En el año 2010 se detectó un inusual aumento de casos de Salmonella en aislamientos de personas, en su mayoría de pacientes pediátricos de diversos hospitales de Lima. El INS identificó 33 aislamientos como Salmonella entérica serovar Infantis, siendo esta la tercera serovariedad más frecuente en el Perú asociado con el consumo de huevos contaminados y a productos cárnicos avícolas ${ }^{(27)}$.

García et al. analizaron 33 aislamientos de Salmonella entérica serovar Typhi provenientes de muestras de sangre (bacteremia) colectada entre el 2008 al 2012 en hospitales de Lima. Las cepas fueron todas sensibles a cotrimoxazol, cloranfenicol, ceftriaxona y tetraciclina. Ocho cepas $(24,2 \%)$ mostraron susceptibilidad intermedia a la ciprofloxacina, presentando mutaciones en gen gyrA (Ser83-Phe yAsp87-Asn) y en el gen gyrB (Ser464-Phe) (28). Por lo tanto, actualmente, la importancia de la Salmonella en nuestro medio radica en la caracterización de brotes y el creciente incremento de la resistencia a quinolonas, antibiótico de elección para este patógeno. Otras drogas de elección para el manejo son ceftriaxona y azitromicina.

En resumen, los patógenos que se han discutido en esta revisión tienen relevancia en la salud pública por su impacto en la carga de enfermedad y mortalidad por diarrea, sobre todo en la población pediátrica. Se requieren estudios nacionales para determinar el impacto de la vacunación contra rotavirus. Además, de deben implementar métodos diagnósticos adecuados en los principales establecimientos de salud del país para lograr una adecuada vigilancia y manejo de las infecciones causadas por estos patógenos (rotavirus, STEC). Debido al incremento en la resistencia antibiótica, sobre todo en Shigella, Salmonella y Campylobacter, se requiere un sistema de vigilancia de la resistencia antibiótica y reforzar el uso racional de antibióticos en médicos del primer nivel de atención.

Contribuciones de autoría: MRR ha participado en la concepción y diseño del artículo, recolección, análisis e interpretación de datos, redacción del artículo y aprobación de su versión final. TOW ha participado en la concepción y diseño del artículo, recolección de resultados, análisis e interpretación de datos, redacción del artículo, revisión crítica del artículo y aprobación de su versión final.

Fuentes de financiamiento: autofinanciado.

Conflictos de interés: los autores declaran no tener conflictos de interés. 


\section{REFERENCIAS BIBLIOGRÁFICAS}

1. Bhutta ZA, Black RE. Global maternal, newborn, and child health--so near and yet so far. N Engl J Med. 2013 Dec 5;369(23):2226-35. doi: 10.1056/ NEJMra1111853.

2. Kotloff KL, Nataro JP, Blackwelder WC, Nasrin D, Farag TH, Panchalingam S, et al. Burden and aetiology of diarrhoeal disease in infants and young children in developing countries (the Global Enteric Multicenter Study, GEMS): a prospective, case-control study. Lancet. 2013 Jul 20;382(9888):209-22. doi: $10.1016 /$ S0140-6736(13)60844-2.

3. Ochoa TJ, Ecker L, Barletta F, Mispireta ML, Gil AI, Contreras $\mathrm{C}$, et al. Age-related susceptibility to infection with diarrheagenic Escherichia coli among infants from Periurban areas in Lima, Peru. Clin Infect Dis. 2009 Dec 1;49(11):1694702. doi: $10.1086 / 648069$.

4. Ochoa TJ, Chea-Woo E, Baiocchi N, Pecho I, Campos M, Prada A, et al. Randomized double-blind controlled trial of bovine lactoferrin for prevention of diarrhea in children. J Pediatr. 2013 Feb;162(2):349-56. doi: 10.1016/j. jpeds.2012.07.043.

5. Ehrenkranz P, Lanata CF, Penny ME, Salazar-Lindo E, Glass RI. Rotavirus diarrhea disease burden in Peru: the need for a rotavirus vaccine and its potential cost savings. Rev Panam Salud Publica. 2001 Oct;10(4):240-8.

6. Clark AD, Walker DG, Mosqueira NR, Penny ME, Lanata CF, Fox-Rushby J, et al. Cost-effectiveness of rotavirus vaccination in Peru. J Infect Dis. 2009 Nov 1;200 Suppl 1:S114-24. doi: 10.1086/605043.

7. Chang MR, Velapatiño G, Campos M, Chea-Woo E, Baiocchi N, Cleary T, et al. Rotavirus Seasonal Distribution and Prevalence Before and After the Introduction of Rotavirus Vaccine in a PeriUrban Community of Lima, Peru. Am J Trop Med Hyg. 2015 Mar 16. pii: 14-0431.

8. Bucher A, Rivara G, Briceño D, Huicho L. Uso de una prueba rápida de rotavirus en la prescripción de antibióticos en diarrea aguda pediátrica: un estudio observacional, aleatorizado y controlado. Rev Gastroenterol Peru. 2012 Jan-Mar;32(1):11-5.
9. Ochoa TJ, Zea-Vera A, Bautista R, Davila C, Salazar JA, Bazán C, et al. Vaccine schedule compliance among very low birth weight infants in Lima, Peru. Vaccine. 2015 Jan 3;33(2):354-8. doi: 10.1016/j.vaccine.2014.11.014.

10. Saito M, Goel-Apaza S, Espetia S, Velasquez D, Cabrera L, Loli S, et al. Multiple norovirus infections in a birth cohort in a Peruvian Periurban community. Clin Infect Dis. 2014 Feb;58(4):483-91.

11. Rivera FP, Ochoa TJ, Ruiz J, Medina AM, Ecker L, Mercado E, et al. Norovirus prevalence in 'pathogen negative' gastroenteritis in children from periurban areas in Lima, Peru. Trans R Soc Trop Med Hyg. 2011 Dec;105(12):734-6. doi: 10.1016/j. trstmh.2011.08.009.

12. Zambruni M, Luna G, Silva M, Bausch D, Rivera F, Velapatino G, et al. High Prevalence and Increased Severity of Norovirus Mixed Infections Among Children 12-24 Months of Age Living in the Suburban Areas of Lima, Peru. J Ped Infect Dis. 2015 Feb 4. doi:10.1093/jpids/piv001.

13. Contreras CA, Ochoa TJ, Ruiz J, Lacher DW, Rivera FP, Saenz Y, et al. Phylogenetic relationships of Shiga toxin-producing Escherichia coli isolated from Peruvian children. J Med Microbiol. 2011 May;60(Pt 5):639-46. doi: 10.1099/jmm.0.026666-0.

14. Llanos A, Lee J, López F, Contreras C, Barletta F, Chea-Woo E, et al. Shiga toxin-producing Escherichia coli in Peruvian children with bloody diarrhea. Pediatr Infect Dis J. 2012 Mar; 31(3):314-6. doi: 10.1097/ INF.0b013e318244000c.

15. Rivera FP, Sotelo E, Morales I, Menacho F, Medina AM, Evaristo R, et al. Short communication: Detection of Shiga toxin-producing Escherichia coli (STEC) in healthy cattle and pigs in Lima, Peru. J Dairy Sci. 2012 Mar;95(3):1166-9. doi: 10.3168/ jds.2011-4662.

16. Mora A, León SL, Blanco M, Blanco JE, López C, Dahbi G, et al. Phage types, virulence genes and PFGE profiles of Shiga toxin-producing Escherichia coli O157:H7 isolated from raw beef, soft cheese and vegetables in Lima (Peru).
Int J Food Microbiol. 20072007 Mar 10;114(2):204-10.

17. Yori PP, Lee G, Olórtegui MP, Chávez CB, Flores JT, Vasquez AO, et al. Santa Clara de Nanay: the MAL-ED cohort in Peru. Clin Infect Dis. 2014 Nov 1;59 Suppl 4:S310-6. doi: 10.1093/cid/ ciu460.

18. Kosek M, Yori PP, Pan WK, Olortegui MP, Gilman RH, Perez J, et al. Epidemiology of highly endemic multiply antibiotic-resistant shigellosis in children in the Peruvian Amazon. Pediatrics. 2008 Sep;122(3):e541-9. doi: 10.1542/peds.2008-0458.

19. Lluque A. Detección de genes de virulencia y resistencia antimicrobiana en Shigella spp. Aislada de niños sanos y con diarrea del distrito de IndependenciaLima. Tesis para obtener el grado de Magister en Microbilogía. Facultad de Farmacia y Bioquímica, Universidad Nacional Mayor de San Marcos. Lima, Perú. 2011

20. Perú, Ministerio de Salud, Proyecto Vigía. Estudio de etiología de la diarrea en las direcciones de salud Cajamarca, Lambayeque, Loreto y Lima Este. Informe técnico [Internet]. Lima: MINSA; 2001 [citado el 09 de febrero de 2015]. Disponible en: http://www. ins.gob.pe/repositorioaps/0/4/jer/ cnsp_resanti_documentos_tecnicos/ Estudio_etiologico_diarrea_4 DISAS.pdf

21. Llancce L. Estudio de resistencia in vitro de cepas de Shigella frente a 20 antimicrobianos en el Hospital Nacional Daniel Alcides Carrión 1999-2001. Tesis para obtener el grado de Especialista en Patología Clínica. Faculta de Medicina, Universidad Mayor de San Marcos. Lima, Perú. 2002

22. Gonzales-Escalante E, Sevilla-Andrade R, León-Sandoval S. B-lactamasas de espectro extendido tipo CTX-M en aislamientos de Shigella flexneri de pacientes pediátricos con diarrea aguda. Rev Peru Med Exp Salud Publica. 2013 Jul-Set;30(3):527-8.

23. Barletta F, Mercado EH, Lluque A, Ruiz J, Cleary TG, Ochoa TJ. Multiplex real-time PCR for detection of Campylobacter, Salmonella, and Shigella. J Clin Microbiol. 2013 
Sep;51(9):2822-9. doi: 10.1128/ JCM.01397-13.

24. Majowicz SE, Musto J, Scallan E, Angulo FJ, Kirk M, O'Brien SJ, et al. The global burden of nontyphoidal Salmonella gastroenteritis. Clin Infect Dis. 2010 Mar 15;50(6):882-9. doi: 10.1086/650733.

25. Arias I, Zamudio M, Luna M, Valenzuela A, Segovia E, Villanueva E. Uso de PFGE en la investigación de brote por Salmonella enteritis en la localidad de Inahuaya, Región Loreto. 2006 Perú. Bol-Inst Nac Salud. 2008 SetOct;14(9-10):197-8.
26. Del Pozo L, Silva N, Valencia A, Soto J, Riveros J, Sacsaquispe R, et al. Estudio de un brote intrahospitalario por Salmonella typhimurium productora de beta-lactamasa de espectro extendido SHV-5.An Fac Med Lima. 2006;67(4):318-26.

27. Zamudio M, Meza A, Bailón H, Martinez-Urtaza J, Campos J. Experiencias en la vigilancia epidemiológica de agentes patógenos transmitidos por alimentos a través de electroforesis en campo pulsado (PFGE) en el Perú. Rev Peru Med Exp Salud Publica. 2011;28(1):128-35.

28. García C, Lejon V, Horna G,
Astocondor L, Vanhoof R, Bertrand $S$, et al. Intermediate susceptibility to ciprofloxacin among Salmonella entericaserovar Typhi isolates in Lima, Peru. J Clin Microbiol. 2014 Mar;52(3):968-70.

Correspondencia: Theresa J. Ochoa Woodell Dirección: Instituto de Medicina Tropical Alexander von Humboldt, Universidad

Peruana Cayetano Heredia, Av. Honorio

Delgado 430, San Martín de Porras, Lima 33, Perú

Teléfono: (+511) 4823903

Correo electrónico: theresa.ochoa@upch.pe

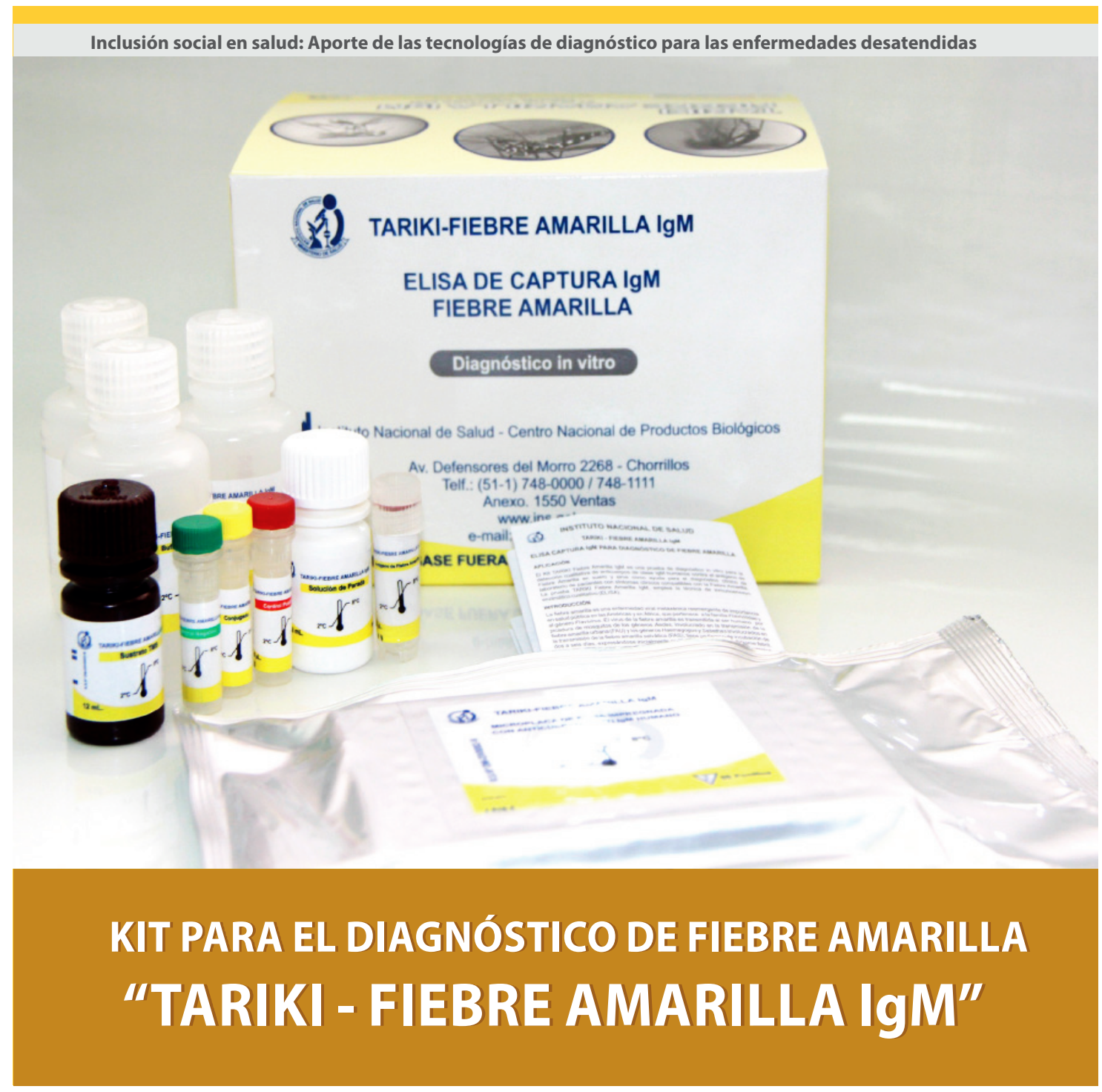

